Cita bibliográfica: Del Alcázar Indarte, H. y Ferrandis Martínez, F. (2021). La revitalización urbanística de los destinos turísticos del litoral: Una alternativa para los municipios turísticos mediterráneos valencianos. Investigaciones Turísticas (22), pp. 354-376. https://doi.org/10.14198/INTURI2021.22.15

\title{
La revitalización urbanística de los destinos turísticos del litoral: Una alternativa para los municipios turísticos mediterráneos valencianos
}

The urban revitalization of the coastal tourist destinations: An alternative for the Valencian Mediterranean tourist municipalities

Héctor Del Alcázar Indarte (iD, Universidad de Valencia, España hector.alcazar@uv.es

Adrián Ferrandis Martínez (iD, Universidad de Valencia, España adrian.ferrandis@uv.es

\section{RESUMEN}

Los municipios del litoral mediterráneo valenciano aumentaron sensiblemente su superficie construida por la irrupción de la actividad turística a mediados del siglo XX. A pesar de las ventajas iniciales, los problemas surgidos sobre el espacio urbano, por la naturaleza del modelo de desarrollo turístico utilizado, los dirigen hacia una pérdida progresiva de su atractivo turístico. Ante esta circunstancia, se hace necesario intervenir sobre el espacio urbano, no sólo para renovar el destino turístico sino también para corregir los problemas urbanos generados durante las últimas décadas en unos municipios que supeditaron sus decisiones exclusivamente a las necesidades turísticas. Una opción viable y más sostenible, a medio y largo plazo, implica cambiar el modelo de desarrollo, tomando como referencia las características de la ciudad tradicional. Sin embargo, la viabilidad y el grado de consecución de esta transformación estará en función de las particularidades del espacio urbano existente en cada caso. El resultado dependerá del modelo de ciudad (Pública o Doméstica) tomado como referencia para su desarrollo y de las características urbanas (Compacta, Mixta o Dispersa) que le sean propias.

Palabras clave: municipios turísticos; transformación urbana; revitalización urbana sostenible; hacer ciudad, renovación turística.

\section{ABSTRACT}

The municipalities on the Valencian Mediterranean coast expanded their urban area in response to the emergence of tourism in the middle of the twentieth century. Despite the initial advantages, the problems that have arisen in the urban space, due to the tourism development 
model used, have led them towards a progressive loss of tourist attractiveness during their different stages of evolution. Given this circumstance, it is necessary to intervene in the urban space, not only to renew the tourist destination but also to correct the urban problems generated over recent decades in some municipalities that subordinated their decisions exclusively to the tourist needs. A viable and more sustainable option, in the medium and long term, implies changing the development model, taking as a reference the characteristics of the traditional city. However, the viability and the degree of achievement of this transformation will be based on the individual characteristics of the existing urban space in each case. Results will be based on the city model (Public or Domestic) used as reference for its development and on the urban characteristics (Compact, Mixed or Dispersed) that are specific to it.

Keywords: tourist municipalities; urban transformation; sustainable urban revitalization; make city; tourist renovation.

\section{INTRODUCCIÓN Y OBJETIVOS DE LA INVESTIGACIÓN}

La irrupción del turismo en los espacios costeros valencianos a mediados del siglo $X X$, fue un fenómeno que quebró el crecimiento lineal y contenido de los pequeños núcleos urbanos diseminados por el espacio rural litoral, en emplazamientos "descubiertos" para el descanso y el ocio, generando una gran expansión de lo urbanizado. En estos municipios se produjo un gran contraste entre la zona preexistente y la nueva zona urbanizada promovida por una actividad económica que crecía de forma exponencial (Sánchez Cabrera, 2011).

Estos núcleos urbanos, en la mayoría tienen su origen en los municipios rurales vinculados con la costa, los cuales podían surgir en contacto con el mar o fundarse en el interior para una mayor protección frente a los enemigos que podían venir por el mar y para estar próximos a las materias primas. En el primer caso, de forma general, su crecimiento se ha producido vinculado al núcleo existente, extendiéndose principalmente de modo paralelo a la línea de costa, mediante la prolongación de su trama urbana. En el segundo caso, el más habitual, la expansión motivada por el turismo se realizó en dos modalidades: vinculada con el núcleo histórico o mediante núcleos urbanos de nueva creación que surgen sin continuidad con el existente, pero en contacto con el mar. En este segundo caso, puede ser que, debido a la importancia histórica del municipio, ya tuviera algún núcleo disgregado en contacto con el mar que es el primero en expandirse, aunque también es posible que, aunque así fuera, se fomenten nuevos núcleos separados de aquél (Barragán Muñoz, 1993; Vera Rebollo e Ivars, 2002; Busquets, 2004).

Teniendo en cuenta este enfoque inicial, la investigación que se presenta en este artículo se plantea como principal objetivo proponer una alternativa de transformación de las ciudades turísticas del mediterráneo valenciano que evolucionaron a partir de los núcleos rurales costeros indicados, con la finalidad de intentar conseguir ciudades más complejas que se enmarquen en mayor medida en el ámbito de la sostenibilidad.

Para alcanzar este objetivo, a partir de un planteamiento de base teórica, en primer lugar, se centra la mirada en entender cuál ha sido el proceso de transformación de los 
municipios rurales en destinos turísticos, su evolución posterior y cuáles eran los problemas más comunes que les afectaban. En segundo lugar, se trabaja en la búsqueda de patrones o modelos de análisis de las formaciones urbanas desde un punto de vista integral, de tal manera que se puedan utilizar para la construcción de la alternativa de renovación de estas ciudades turísticas. En tercer lugar, se estudia la viabilidad y las posibilidades de alcanzar los parámetros urbanos de referencia, tomando como punto de partida la diversidad de situaciones existentes. Y, por último, en cuarto lugar, se elaboran unas conclusiones centradas en identificar las limitaciones existentes y las problemáticas que podrían encontrar en la aplicación de las propuestas, a pesar de la convicción de su validez.

\section{LA SITUACIÓN EN LA QUE SE ENCUENTRAN LOS MUNICIPIOS TURÍSTICOS}

El turismo tuvo una gran influencia sobre el crecimiento y la evolución de los municipios situados en el litoral pues se convirtió en la actividad económica motora al substituir, por su mayor rentabilidad, a las actividades económicas tradicionales que estaban vinculadas mayoritariamente con el sector primario (Barragán Muñoz, 1993; Aledo, 2008). En núcleos urbanos de mayor entidad que habían ido evolucionando gradualmente a lo largo del tiempo, la irrupción del turismo también tuvo una gran influencia, pero al contar con un modelo económico diversificado, éste se convirtió en una actividad que complementó a las previamente existentes. Además, en estos casos, las ciudades no necesitan supeditarse a las exigencias de la demanda turística para obtener la viabilidad económica y una buena calidad de vida para sus habitantes. Por esta razón, la expresión territorial de los nuevos desarrollos ligados al turismo en las ciudades, es más contenida en comparación con el espacio urbano preexistente, y puede integrarse mejor con la realidad territorial consolidada.

En un principio, el turismo tuvo unos efectos beneficiosos muy importantes en los municipios turísticos, plasmados en los ingresos que la actividad turística proporcionaba a la administración local a través de los impuestos y las tasas; en las opciones laborales promovidas que permitieron tanto fijar profesionalmente a sus habitantes, como convertirse en un polo de atracción de nueva población; y en el significativo aumento de la oferta socio-cultural que trajo aparejada (Perlado y Elorrieta, 2007; Pitarch, 2009). Sin embargo, el proceso de trasformación y cambio se desvirtuó cuando, ante la incidencia de la nueva actividad económica, se supeditó todo a satisfacer las exigencias de la demanda turística. Este posicionamiento por parte de los distintos niveles de gobierno, especialmente de los locales (con las competencias en materia urbanística), pero con la conveniencia o inhibición del resto, hizo que se perdieran de vista las necesidades básicas de calidad de vida de sus habitantes, la estructura urbana que organizaba el conjunto construido o la relación del núcleo urbano con el entorno circundante, que en muchos casos era el principal reclamo turístico (Callizo Soneiro, 1991; Vera Rebollo, 1996).

Los municipios rurales de pequeño tamaño, en la mayoría de los casos, estaban comandados por una Administración Local que carecía de la experiencia y de las herramientas necesarias para gestionar los enormes cambios urbanos y territoriales promovidos por la actividad turística, lo que hizo que los actores vinculados a este sector (empresas constructoras, inmobiliarias, tour operadores, empresarios del sector turístico, etc.) fueran los que dictaran 
de facto la política urbanística, basada en un crecimiento extensivo y continuo, que se desarrolló dentro de los límites del municipio (Perlado y Elorrieta, 2007). De hecho, la población autóctona, ante las penalidades de las épocas anteriores para subsistir en el territorio, vistos y comprobados los beneficios que observaban aparejados a la irrupción del turismo, así como la proyección de éstos hacia el futuro, no fue consciente, al menos en las primeras etapas (años 60-80 del S. XX), de las posibles consecuencias negativas que, paralelamente, podría también acarrear el desarrollo turístico. Además, si había cualquier conato de discrepancia u objeción ante el modo de proceder, al estar en una dictadura ${ }^{1}$, era fácilmente tapada por la propaganda política y empresarial, cuando no simplemente reprimida. Posteriormente, ya en democracia, se asumió que los inconvenientes estructurales tanto en el núcleo urbano como en el territorio eran el precio que se debía pagar para asegurar el mantenimiento de ese supuesto bienestar conseguido con la nueva actividad económica. Así, muchos municipios rurales pasaron a ser municipios turísticos. $\mathrm{O}$, aún peor, un destino turístico supeditado a satisfacer mayoritariamente el disfrute del visitante (García-Delgado, 2002).

\subsection{Factores que propiciaron la transformación de los municipios rurales situados en el litoral a municipios turísticos}

Existen diversos factores que favorecieron dicha transformación, muchos de los cuales están interrelacionados y se influencian o complementan entre sí. En primer lugar, y aunque es obvio, la propia irrupción del turismo en las zonas litorales, a partir de las primeras décadas del S.XIX, aunque con una mayor intensidad a partir de la década de 1960-70 (Tatjer, 2009). En segundo lugar, el avance social que supuso disponer de tiempo libre conseguido con las vacaciones pagadas, a partir de la Convención de la Organización Internacional del Trabajo celebrada en 1936, que se materializará de forma palpable a partir del fin de la II Guerra Mundial (Callizo Soneiro, 1991; Torres Alfosea, 1997). En tercer lugar, la instrumentalización que el sector inmobiliario hace del turismo, del cual se apropia, para promover la construcción de edificios residenciales y para la venta de sus viviendas y, en consecuencia, la obtención de beneficios para aquellas empresas relacionadas con el sector (constructoras e inmobiliarias) en lugar de para las empresas insertadas en el territorio dedicadas a ofrecer servicios a los turistas (Busquets, 2003; Mantecón, 2005; Fariña Tojo y Naredo, 2010). Dentro de esta dinámica, que ha imperado en las zonas litorales mediterráneas valencianas, especialmente en las primeras décadas de inicio de la actividad, se promovió la continua introducción de terreno edificable, pues era el modo mediante el que estas empresas podían obtener sus beneficios, dejando de lado el objetivo de proporcionar unas condiciones que propiciaran y potenciaran su atractivo turístico (Pié y Barba, 1996; Fernández Tabales, 2004; Mazón y Aledo, 2005). En cuarto lugar, el hecho de que el país se encontraba bajo un régimen dictatorial durante el cual se produjo la aprobación de diversas leyes que favorecieron la transformación del suelo y que tenían, como se ha podido comprobar retrospectivamente, el objetivo de proporcionar las condiciones legales necesarias para que la actividad inmobiliaria se desarrollara más libremente: la Ley del Suelo (1956) de carácter más general y no exclusivamente con finalidades de desarrollo urbano

1. España se convirtió en un país democrático de derecho mediante la ratificación en referéndum de la Constitución Española el 6 de diciembre de 1978. 
turístico, y las Leyes de Centros y Zonas de Interés Turístico Nacional (1963) y de Costas (1969), con una vinculación mucho más clara y propiciadora de transformación de suelo (Calduch, 2001). Una vez ya en democracia, se utilizaron los Planes Generales de Ordenación Urbana (PGOUs), documentos técnicos surgidos ya durante la dictadura, para ordenar y cualificar los crecimientos desmedidos sobre los núcleos urbanos promovidos durante el periodo tardofranquista. Sin embargo, al tratarse de unos documentos complejos y de difícil modificación, éstos no se actualizaron respecto a las necesidades estructurales del municipio, sino que se limitaron a alteraciones puntuales, sin que se planteara el modelo de ciudad que se quería para el futuro (Fariña Tojo y Naredo, 2010; Navarro Jurado, 2012). Posteriormente, con la entrada en vigor en 1994 de la Ley Reguladora de la Actividad Urbanística (LRAU) en la Comunitat Valenciana, ley que sería tomada más adelante como ejemplo para el desarrollo de la legislación urbanística en otras Comunidades Autónomas, abrió la posibilidad a que la ordenación (tanto la territorial como la urbana) pudiera estar gestionada por las administraciones públicas competentes, pero también mediante la gestión privada, con la cesión de competencia a la, en ese momento, novedosa figura del agente urbanizador (Gaja, 2008). Cabe matizar que esta figura, con competencias en la promoción urbanística, estaba pensada originariamente para la creación de espacios industriales ordenados y urbanizados (carentes por esa época), pero que al mismo tiempo facilitó el desarrollo de sectores residenciales y, por supuesto, de actuaciones residenciales turísticas. En quinto lugar, la infrafinanciación de las Administraciones Locales que les impedía atender adecuadamente las competencias propias o sobrevenidas para con sus ciudadanos, lo que les obligó a buscarse mecanismos para la obtención de financiación complementaria. Esta situación se materializó en la recalificación de suelo rural en suelo urbano (Fernández Tabales, 2004; Mazón y Aledo, 2005; Aledo et al., 2007; Aledo, 2008; Cortés Chavez, 2009; Obiol y Pitarch, 2011). En sexto y último lugar, la proliferación y la popularización de la segunda residencia, la cual suele estar vinculada con las zonas recreativas y que tendrán un uso de fin de semana o durante la época de vacaciones, dependiendo de la distancia existente entre ésta y la vivienda habitual (García Nart et al., 1991; Mazón y Aledo, 2005).

Tabla 1: Factores que favorecieron la transformación de municipio rural del litoral a municipio turístico

\begin{tabular}{|c|c|}
\hline Núm. & Factor \\
\hline 1 & $\begin{array}{l}\text { Irrupción del turismo en el litoral levantino (primeras décadas S.XIX, aunque intensificación } \\
\text { en el periodo 1960-70) }\end{array}$ \\
\hline 2 & $\begin{array}{l}\text { Aumento del tiempo libre por la consolidación de las vacaciones pagadas (1936 acuerdo de } \\
\text { la OIT; en España generalización a partir del periodo 1960-70) }\end{array}$ \\
\hline 3 & Instrumentalización del turismo por el sector inmobiliario (periodo 1960-80) \\
\hline 4 & $\begin{array}{l}\text { Aprobación de legislación que favorece la actividad inmobiliaria (Leyes de Centros y Zonas } \\
\text { de Interés Turístico Nacional en } 1963 \text { y de Costas 1969; así como en un periodo posterior, } \\
\text { con la LRAU de la Comunitat Valenciana en 1994). }\end{array}$ \\
\hline 5 & $\begin{array}{l}\text { Infrafinanciación de las Administraciones Locales (Problema persiste desde la época } \\
\text { Franquista hasta la época democrática). }\end{array}$ \\
\hline 6 & Proliferación y popularización de la Segunda Residencia (A partir de los años 1970-80). \\
\hline
\end{tabular}

Elaboración propia. 


\subsection{La evolución de municipio rural del litoral a municipio turístico}

Una vez enumerados los factores que favorecieron dicha transformación conviene atender a las diferentes fases que, con carácter general, atraviesa un destino turístico, y ello con el objetivo de analizar en qué situación se encuentran los distintos municipios para tomar conocimiento de que inconvenientes se han detectado para el futuro y cómo se puede lidiar con ellos.

Existen diversos modelos teóricos que tratan de explicar la evolución de los destinos turísticos. Los tres de referencia básica son: el modelo funcional de Miossec de 1977, el modelo historicista de Chadefaud de 1987 y el modelo del ciclo de vida de Butler de 1980. Todos ellos estructuran la evolución de los destinos turísticos en diferentes fases (Tabla 2) y, a pesar de que cada uno de ellos parte de una concepción teórica diversa, sostienen que todos los destinos llegan a un punto de saturación a partir del cual se ven abocados a replantear y modificar su patrón de desarrollo. De la confrontación de los tres modelos también se desprende que la evolución de un destino turístico está condicionada por tres factores: “(1) los componentes territoriales, económicos y sociales que lo caracterizan, (2) el contexto económico de la región donde se localiza, y (3) las tendencias económicas generales que afectan la demanda y la oferta" (Vera Rebollo et al., 2013, p. 230).

Tabla 2: Fases por las que pasa un destino turístico en función del modelo teórico

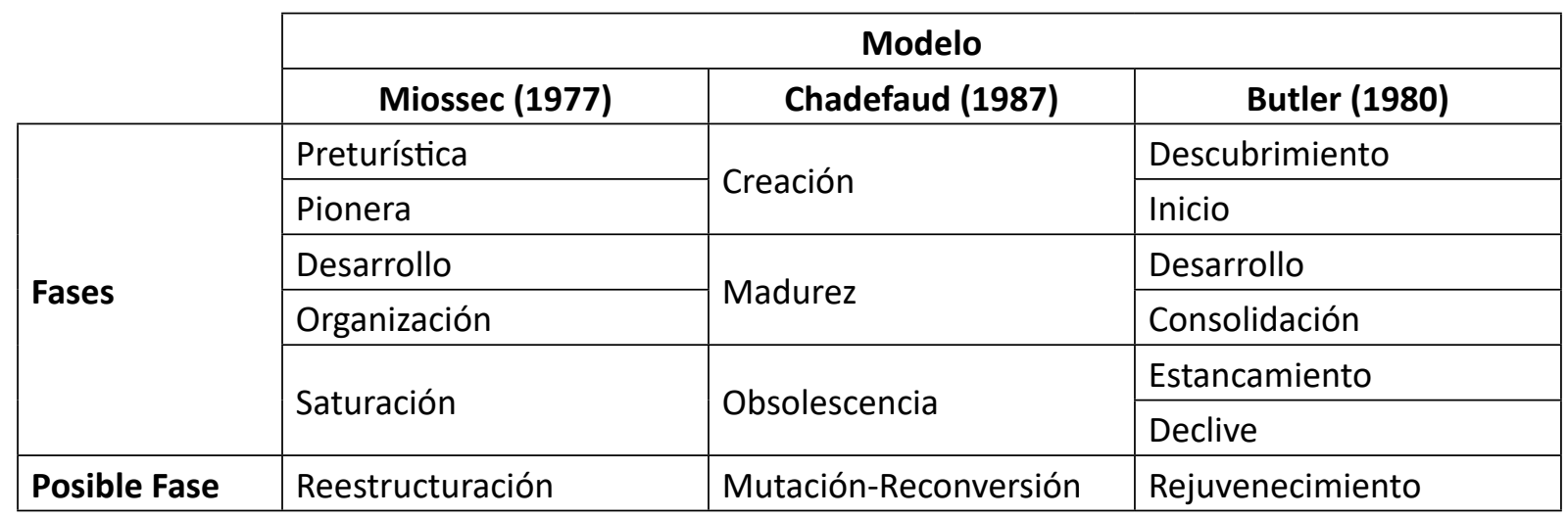

Fuente: Elaboración propia a partir de Vera Rebollo et al. (2013).

De entre todos ellos, consideramos que el modelo de Butler (1980) esboza claramente y de forma sencilla la evolución que han experimentado la mayoría de los destinos turísticos consolidados de "sol y playa" del litoral mediterráneo español. Ese proceso está dividido en seis fases que van desde el descubrimiento de las potencialidades turísticas de un municipio, pasando por el inicio, desarrollo, consolidación y estancamiento, hasta llegar al declive. Según este modelo, el ciclo vital del municipio litoral como polo atrayente de turistas lo encamina inexorablemente, por su propia naturaleza, a la pérdida de atractivo desde el momento en que supera su capacidad de carga, punto en el cual las consecuencias negativas solo pueden ser paliadas con la intervención continua de los agentes implicados en el municipio. Por lo tanto, el modelo urbano y territorial sobre el que se sustenta el desarrollo del municipio turístico lo empuja a su propia destrucción como destino para turistas. Y únicamente se pueden paliar los 
problemas, que no corregir las causas, pues el principal problema es el modelo y, por lo tanto, los resultados que se obtienen más pronto o más tarde son consustanciales a éste (Donaire y Mundet, 2002).

\subsection{Principales problemáticas urbanísticas identificadas en esta tipología de municipios turísticos}

Así pues, el turismo ha generado una nueva realidad en muchos municipios litorales, como sucedió anteriormente en muchas ciudades por la influencia de la industria. Nos encontramos en unas fases ya muy avanzadas de transformación y ocupación de suelo, que, utilizando las terminologías clásicas de la evolución de los destinos turísticos, de forma general, ubican a estos destinos de "sol y playa" (dentro de sus variedades y particularidades concretas) entre las etapas de madurez, saturación o estancamiento, o en una situación intermedia de entre todas ellas (Obiol y Ferrandis, 2011).

Ante esta realidad, se deben proponer actuaciones encaminadas a intentar revertir estas situaciones. A nivel general, las dos principales problemáticas urbanísticas identificadas en estas tipologías de municipios turísticos litorales son: por un lado, que en aquellos casos en que el núcleo histórico se encontraba en el interior del territorio se ha llevado a cabo una gran expansión urbana en la zona rural en contacto con la costa que le ha dado completamente la espalda al núcleo histórico; y por otro lado, que el gran crecimiento urbano ha tenido un fuerte y casi único carácter residencial con el objetivo de generar alojamiento para los turistas (Ferrer Marsal, 2002). Este desarrollo urbano se realiza casi exclusivamente con viviendas de segunda residencia que, en la mayoría de los casos, son utilizadas únicamente durante los fines de semana y las épocas de vacaciones (es decir, durante un tercio del año en el mejor de los casos) por lo que están infrautilizadas (García Nart et al., 1991). Además, este crecimiento no ha asegurado los estándares mínimos de espacio público y dotaciones correspondientes a la población que puede llegar a soportar durante los meses de apogeo turístico (Perlado y Elorrieta, 2007).

Es por ello que se entiende que el modelo que sustenta la mayoría de los municipios turísticos consolidados del litoral Mediterráneo valenciano (extrapolable también a otros municipios del resto de España) presenta determinados síntomas de agotamiento, es un gran consumidor de territorio (con las consecuencias que pueden derivar de ello a nivel medioambiental y paisajístico) y, en general, es poco sostenible especialmente en el medio y largo plazo.

\section{ANÁLISIS DE LA OPCIONES DE INTERVENCIÓN PARA REVITALIZAR LOS MUNICIPIOS TURÍSTICOS COSTEROS}

Existen diferentes posicionamientos al respecto de cómo actuar en esta tipología de municipios turísticos con el objetivo de intentar revitalizar sus tramas urbanas y generar espacios más atractivos, habitables y, como no puede ser de otra manera, más sostenibles, tanto para sus residentes estacionales, como también para los permanentes. Desde el ámbito de la revisión de los planteamientos teóricos, base fundamental de la presente propuesta y, a modo de síntesis, se puede decir que existen tres opciones de intervención: en primer lugar, apostar 
por el mantenimiento del modelo y la consolidación de la función residencial en el municipio turístico que es la que ha condicionado la estructura urbana y la morfología actual; en segundo lugar, destruir buena parte de lo construido y regenerar el territorio para devolverlo a la situación previa a la irrupción del turismo y el boom inmobiliario aparejado; y en tercer y último lugar, intentar dotar de la calidad suficiente y transformar dichos municipios en ciudades y en núcleos más potentes dentro de un área territorial que abarque diversos municipios vecinos.

La primera opción, aunque existen ciertos sectores económicos (negacionistas de las bondades del Desarrollo Sostenible) que la defiendan como una opción licita para seguir generando riqueza, no se puede contemplar como una solución a tener en cuenta porque, al no asumir los problemas generados por el desarrollo urbano no planificado llevado a cabo en estos municipios litorales, no le puede dar solución tal y como defienden autores de referencias como Pujadas y Font (1998), Folch (2003), Esteban (2006), Farinós (2006), Gómez Orea (2007) o el propio Libro Blanco de la Sostenibilidad en el Planeamiento Urbanístico Español (2010) entre otros... (Ferrandis y Noguera, 2016).

La segunda vía resulta prácticamente inaplicable por el coste económico que podría implicar, los recursos materiales y de todo tipo ya consumidos de partida, por los aspectos sociales y culturales generados a lo largo de su historia, así como por razones de tipo jurídico (Gaja, 2012). Así pues, cabe decantarse por la tercera solución y postularse por la necesidad de intentar regenerar estos núcleos urbanos. Sin embargo, para iniciar un proceso de estas características, se debe partir de dos condicionantes importantes: por una parte, las limitaciones de la actuación de regeneración urbana al ámbito construido existente (Santatecla, 2015; Tuset y Temes, 2015); y por otra parte, la substitución, a partir de metodologías basadas en la transferencia de aprovechamientos, de aquellos edificios residenciales más propicios por elementos de los que se carece en el núcleo urbano (espacio público, zonas verdes, equipamientos públicos, comercio de barrio/proximidad...etc.), aunque sin perder de vista los derechos consolidados de los propietarios, que en algunas ocasiones pueden resultar de difícil reconversión.

Ambas condiciones están interrelacionadas. Por un lado, si no limitáramos el ámbito de actuación a la zona ya construida, la solución más fácil y menos contestada por los intereses privados sería la utilización del espacio periurbano para acoger las carencias detectadas, lo que generaría un aumento del espacio urbanizado y la consolidación de una estructura de espacio público deficiente que no vertebraría el espacio construido existente y que está detrás de gran parte de los problemas que arrastran estos municipios. Por otro lado, si se restringe la actuación a la zona construida, si no se eliminan o se substituyen algunos de los edificios existentes, no se podrá generar una estructura de espacio público que la vertebre ni se tendrán lugares donde ubicar los equipamientos y los usos necesarios para la vida cotidiana. A pesar de esto, estos condicionantes no impiden las intervenciones en los bordes urbanos, sino que promueven que éstas estén enfocadas a introducir lo rural en lo urbano en lugar de lo inverso.

Así pues, para transformar estos municipios turísticos en ciudades (hacer ciudad), será necesario conocer una serie de características que las identifican (Concepto Ciudad): (1) disponer de espacio público que vertebre y ordene el núcleo urbano en las distintas escalas urbanas (manzana, barrio y ciudad), el cual sea un espacio de estancia y de relación donde se facilite el 
encuentro, el intercambio y la mezcla y cohesión de diferentes grupos sociales y culturales; y en el que se acoja una gran variedad de usos (Borja y Muxí, 2000; Perlado y Elorrieta, 2007); (2) ofrecer unos servicios mínimos (alcantarillado, suministro de agua, etc.) y los equipamientos necesarios para la vida cotidiana (institucionales, educativos, sanitarios, culturales, religiosos, deportivos y de ocio); (3) disponer de variedad de usos del suelo (residencial, comercial, dotacional y terciario) repartidos por la trama urbana; (4) contar con elementos de centralidad (edificios dotacionales y espacios públicos abiertos) que ordenen y jerarquicen la estructura urbana; (5) que el ciudadano sea la unidad de medida en las diferentes escalas de la urbe; (6) que exista una red de transporte público que conecte los distintos barrios; y (7) que el peatón sea el protagonista del espacio público (Oliva i Casas, 2006). No se debe olvidar, que revitalizar un espacio urbano y, especialmente los de uso turístico, implica también mirar por crear espacios lo más sostenibles posibles, por lo que estas últimas actuaciones enmarcadas en mejorar la movilidad sostenible, resultan igualmente características fundamentales.

En línea con este último punto de vista, caminar hacia un urbanismo más sostenible y, en definitiva, hacia la consecución de unas ciudades turísticas sostenibles, pasa ineludiblemente por mejorar los parámetros de accesibilidad y movilidad desde un planteamiento integral. En este sentido, resulta también muy recomendable a la hora de intentar hacer ciudad, tener en cuenta los planteamientos del llamado urbanismo ecológico (Rueda et al., 2012). Según este posicionamiento, se debe considerar la idoneidad de los desarrollos urbanísticos en función de las características del emplazamiento y de las potencialidades en cuanto a la consecución de la habitabilidad urbana y de la eficiencia del sistema urbano. Dicha eficiencia pasa también por aportar nuevas estructuras morfológicas urbanas que propicien la movilidad y la accesibilidad, especialmente del peatón, principal protagonista del espacio público, tal y como ya se ha indicado (Oliva i Casas, 2006). Para poder alcanzar estos planteamientos, según Rueda et al. (2012), es necesario diseñar células urbanas (manzanas) que creen espacios libres de flujos motorizados, dando paso a albergar el resto usos (plazas públicas, zonas verdes, equipamientos necesarios para la vida cotidiana, espacios dotacionales para prestación de servicios, comercios de barrio/proximidad... etc.) y en los que el peatón resulte el agente principal.

Teniendo en cuenta todos estos planteamientos, para intentar regenerar estos núcleos urbanos turísticos, una de las cosas que se debe hacer es aceptar el crecimiento y la evolución morfológica que los municipios litorales han experimentado en un periodo de tiempo muy breve (los últimos sesenta o setenta años), en comparación con el tiempo transcurrido desde su fundación, para tener el núcleo urbano de partida sobre el cual intervenir corrigiendo los problemas generados por el modelo turístico sustentado en el residencialismo; es decir, la construcción de viviendas destinadas a segunda residencia o a generar plazas de alquiler turístico (Tuset y Temes, 2015). Posteriormente, las intervenciones deben intentar estar guiadas por dos premisas: promover un crecimiento interno, no expansivo, y asegurar la presencia de las características de lo que hemos denominado hacer ciudad o Concepto de Ciudad. En estas premisas subyace el concepto de "construir sobre lo construido" que defiende que las transformaciones y mejoras urbanas necesarias para aproximarse al Concepto de Ciudad se deben realizar sobre el tejido urbano, haciendo el mínimo uso posible del espacio periurbano que, aunque influido por estar en contacto con lo urbano, haya conseguido mantenerse sin urbanizar. Es aquí donde entran los procesos urbanísticamente de reforma interior, no exentos de 
problemáticas y conflictos sociales y económicos. La forma más factible de poder desarrollarlos pasa por aplicar las indicadas transferencias de aprovechamiento en otras parcelas, bien mediante la ampliación en altura sobre manzanas próximas (técnica igualmente problemática, sobre todo si ya poseen de edificaciones preexistentes, como es de suponer para estos casos), o bien en parcelas de crecimientos urbanísticos ya previstos y compactos a la trama urbana existente (mediante la compensación de las unidades de aprovechamiento correspondientes al desfase de valor entre la nueva ubicación y la inicial).

Al hablar de las posibilidades de intervención en los municipios costeros del litoral valenciano, no se puede obviar el hacer referencia a los instrumentos de planeamiento territorial desarrollados recientemente. Tomando como base los principios establecidos en la Estrategia Territorial de la Comunitat Valenciana de 2011, y su posterior transposición en la Ley de Ordenación del Territorio, Urbanismo y Paisaje (LOTUP) de 2014², en su artículo 16, se habilita la competencia de los Planes de Acción Territorial (PAT) de reclasificar suelo, independientemente de su tipología y calificación. A partir de esta competencia, se ha elaborado el Plan de Acción Territorial de la Infraestructura Verde del Litoral (PATIVEL) de 2018, cuyo principal objetivo es actuar sobre los espacios costeros, intentando preservar el conjunto de espacios abiertos libres de edificación (a modo de ventanas abiertas al mar), mediante la aplicación de diferentes niveles de protección, de tal manera que se evite su trasformación a suelo urbano, estableciendo un límite claro en la trama urbana de los municipios turísticos del litoral. Sin duda alguna, este tipo de actuaciones vinculadas al planeamiento territorial, favorecen y priorizan las intervenciones urbanas en los espacios interiores de las ciudades, de tal forma que pueden propiciar la regeneración de los espacios urbanos objeto de la presente propuesta.

Tomando en consideración todo lo argumentado hasta este punto, y ante la apuesta por el planteamiento de la intervención sobre los núcleos urbanos litorales para dotarlos de las características del Concepto de Ciudad (sostenible, con espacios públicos, zonas verdes, dotaciones, mezcla de usos, protagonismo del peatón... etc.), se puede optar básicamente por dos opciones: por una parte, transformarlos en ciudades turísticas, que -por ejemplo- y a pesar de su singularidad, es el camino que tomó en su día Benidorm ${ }^{3}$, mediante la tematización y la ultra-especialización para dar respuesta a las necesidades de ocio y descanso de los visitantes; y por otra parte, transformarlos en ciudades "sin adjetivo", en artefactos urbanos complejos en los cuales se persigue la atracción de residentes permanentes y la introducción y consolidación de las actividades económicas propias de la ciudad, con el objetivo de diversificarlas (Pié, 2005). La primera opción implica poner el foco en satisfacer las demandas de los turistas, mientras que la segunda lo pone en las necesidades de los residentes (con sus

2. Recientemente revisada por la Ley $1 / 2019$ de modificación de la Ordenación del Territorio, Urbanismo y Paisaje (LOTUP) de 2014. Modifica, añade y corrige errores ortográficos de distintos artículos.

3. Benidorm representa un caso único y singular en el marco del territorio valenciano y español. La ciudad de Benidorm se caracteriza por una complejidad de funcionalidades urbanas muy distintas al del resto de ciudades turísticas del litoral valenciano (Iribas, 1997). Por resaltar algún dato, la ciudad destaca por su amplia oferta de plazas en hoteles, 43.335 (INE, 2018), y un grado de ocupación media anual superior al 80\% en hoteles y al $70 \%$ en apartamentos. 
complejidades, por las necesidades de los residentes, especialmente en lo que se refiere a la esfera socioeconómica, como, por ejemplo, en el ámbito laboral... etc.).

Sin embargo, esta primera opción únicamente puede ser llevada a cabo en unos pocos casos, ya que los municipios que optan por esta solución competirían tanto con otros municipios que apostaran por introducir las características de complejidad de la ciudad, como con los que únicamente son un destino turístico, lo que supondría enormes dificultades para sostener el modelo en el tiempo. Es por ello que se propone, con carácter general, la alternativa de la segunda opción: intervenir en el municipio turístico para que adquiera características lo más próximas posibles a las de una ciudad, a pesar de los limitantes existentes y las particularidades de cada destino (Pié, 2005; Cals y Bosch, 2005), promoviendo la introducción y la potenciación de la diversidad de actividades económicas que en ella son características, sin olvidar ni dejar de lado que la actividad turística ha sido el motor de su gran desarrollo (Rovira Soto y Anton Clavé, 2014).

\subsection{El cambio de modelo: de municipio turístico a ciudad}

Así pues, si se asume que el modelo turístico característico del Mediterráneo valenciano ( $y$, en parte, también del español), el cual está sustentado mayoritariamente en el residencialismo, presenta problemáticas de viabilidad en el medio y largo plazo, con lo que precisa de su reconversión ante las nuevas demandas turísticas centradas en el disfrute del medio ambiente, la oferta cultural diversificada y de calidad y la búsqueda de experiencias que transmitan autenticidad y originalidad (Ball-llosera y Albó, 2005), se hace imprescindible la puesta en valor del hecho urbano como producto turístico y, en consecuencia, el intento de cambio de modelo como alternativa de futuro para el desarrollo de estos núcleos urbanos.

Para llevar a cabo esta transformación habrá que proveer al municipio turístico, mediante las intervenciones oportunas, de las características descritas del Concepto de Ciudad. Con la finalidad de conseguir este objetivo, dichas características se deben cumplir en las diferentes escalas de lo urbano: tanto en el conjunto de la urbe, como en los diferentes barrios en los que está estructurada. Dada la complejidad de esta propuesta de intervención se hace necesario realizar una planificación estratégica a medio y largo plazo, e intervenir sobre lo construido. Sin embargo, para que estas acciones de plasmación física sobre el espacio urbano sean factibles y efectivas, deben ir acompañadas de actuaciones que las secunden en otros planos (social, legislativo, político, turístico, etc.) como, por ejemplo: la conformación de una unidad supramunicipal para la toma de decisiones de ámbito territorial; la promoción de nuevas actividades económicas que diversifiquen al turismo; la utilización de instrumentos normativos y políticas fiscales que incentiven y promuevan las actuaciones urbanas deseadas; la búsqueda de nuevos modos de financiación local desligados de la construcción; la ejecución de políticas de movilidad urbana que beneficien al peatón y al transporte colectivo; la mejora del funcionamiento del metabolismo urbano en especial en la mejora de la eficiencia en el consumo de agua y energía; el cuidado por preservar y enriquecer al tejido social existente o la colaboración público-privada para la mejora y actualización del sector turístico. 
En ambas soluciones planteadas, la de hacer que el municipio turístico evolucione para convertirse en una ciudad turística y la de convertirlo en una ciudad "sin adjetivo", habrá que llevar a cabo intervenciones enfocadas, por un lado, a renovar y restructurar el municipio como destino turístico (Blanco, 2012) y, por otro lado, a hacer ciudad (Barragán Muñoz, 1993; Pié, 2005; Donaire, 2005; Cals y Bosch, 2005; Simancas, 2012; Tuset y Temes, 2015). Es decir, hay que valorar y mantener la actividad económica que ha impulsado el desarrollo urbano de estos municipios, pero emprendiendo un cambio de modelo en el que se inserten intervenciones coordinadas que mejoren el espacio urbano existente y las condiciones de vida de los residentes.

Para llevar a cabo la renovación como destino turístico se requiere "la integración coordinada de procesos de renovación urbanística y gestión ambiental y paisajística, junto con acciones orientadas a la modernización y cambios en el tipo de establecimientos y servicios de turismo y ocio" (Vera Rebollo y Baños Castiñeira, 2010, pp. 349-350), con el añadido de propuestas que motiven su desestacionalización (Barragán Muñoz, 1993; Cals y Bosch, 2005). La renovación del destino debe estar liderada por el sector hotelero o, en el caso de que éste sea débil, por la administración local (Pié y Barba, 1996), y debe basarse en un plan estratégico de posicionamiento turístico previamente consensuado entre todos los agentes locales (instituciones, empresarios y ciudadanía) (Perelli, 2012). Sin embargo, cabe destacar que no todos los destinos turísticos son susceptibles de renovarse pues para ello se requiere: (1) una alta presencia de empresas del sector turístico, (2) un diagnóstico compartido, (3) el convencimiento generalizado de la necesidad de transformación del destino y (4) un liderazgo político que permita alcanzar los objetivos a medio y largo plazo (Perelli, 2012). Estas características, sin embargo, no son muy comunes en esta tipología de municipios turísticos que, salvo honrosas excepciones, no han basado únicamente su economía en la actividad turística sino también en la construcción de viviendas para turistas.

Es preciso enfatizar que la renovación no debe llevarse a cabo mediante la construcción de nuevas infraestructuras de transporte en el territorio, sino fomentando una utilización de las ya existentes de un modo más eficiente (Pié et al., 2005). La experiencia ha demostrado que las mejoras en la accesibilidad actúan como efecto llamada, generando más tráfico que se expande por todas aquellas vías que lo permitan. Es por ello que se hace indispensable jerarquizar la red viaria y que no se dimensione ésta en función de la demanda máxima puntual de movilidad (Cals y Bosch, 2005).

Estos procesos o acciones enfocados a renovar el destino turístico se pueden agrupar en cinco bloques de actuación: en primer lugar, intervenir en el paseo marítimo y en el espacio de conexión entre la costa y la ciudad; en segundo lugar, mejorar los servicios ofertados en las playas y el puerto, de tenerlo; en tercer lugar, peatonalizar las zonas urbanas que iría unida a la puesta en marcha de un espacio comercial abierto; en cuarto lugar, realizar esponjamientos para reducir la compacidad urbana, en aquellos casos en los que resulte excesiva, mediante la posible demolición de edificios de apartamentos, con la problemática que ello puede implicar; y en quinto lugar, actualizar el mobiliario urbano y su homogeneización con la intención de transmitir una identidad unitaria y fácilmente reconocible (Priestley, 1996; Donaire y Mundet, 2002; Pié, 2005; Simancas, 2012). Respecto a las edificaciones relacionadas con el turismo, se 
deben reservar los mejores emplazamientos para los hoteles y facilitar que el parque existente se reforme y se adecue a las demandas actuales. Por lo que hace a la oferta extra hotelera, es necesario controlar y restringir la oferta y exigir que ofrezca la misma calidad que deben cumplir los hoteles (Pié, 2005). Sin embargo, esta propuesta de promover el turismo mediante la reserva de los enclaves litorales más atractivos para el alojamiento del habitante temporal impide que el residente pueda vivir en dichos emplazamientos (Chiner Vives, 2006).

En cuanto a la idea de hacer ciudad, se tendría que intentar subsanar los dos principales problemas que se encuentran en los municipios litorales: la ausencia de residentes durante grandes periodos del año y la falta de dotaciones y servicios necesarios para la vida cotidiana. Por ello, las actuaciones deberían ir encaminadas a: en primer lugar, promover un centro urbano que articule de forma más coherente la trama urbana y que dote de identidad al espacio construido; en segundo lugar, gestionar un patrimonio inmobiliario sobredimensionado, generalmente usado como segunda residencia, y en muchos casos de mala calidad, pues está proyectado pensando únicamente en un uso veraniego, para su reconversión, en parte, en primera residencia en todos los casos que existieran las condiciones que lo permitiera y fomentando la mezcla de usos de primera y segunda residencia allí donde resulte compatible; en tercer lugar, dotar de los equipamientos y servicios necesarios para la vida diaria de los que se carece, que deben estar más enfocados al residente permanente que al turista aunque beneficien a ambos; y en cuarto lugar, potenciar la atracción de empresas innovadoras que contribuyan a diversificar la actividad económica y a incrementar las posibilidades de ofrecer empleos del mercado de trabajo local (Blanquer et al., 1983; Donaire y Mundet, 2002; Esteban et al., 2005; Pié, 2005; Pié et al., 2005; Cals y Bosch, 2005; Fariña Tojo y Naredo, 2010; Obiol y Pitarch, 2011; Santatecla, 2015; Tuset y Temes, 2015). Un posible ejemplo de este tipo de intervenciones, a pesar de que no tuvo el éxito esperado, es el municipio de Calvià, en el cual, mediante el impulso que supuso la aprobación de la Agenda 21 Local en 1996, se pudieron tramitar distintas modificación del planeamiento urbanístico que consiguieron desclasificar suelo urbanizable (hasta 1.350 ha.), cancelar la ejecución de 40.000 plazas hoteleras y llevar a cabo un plan de esponjamiento con el cual se derribaron doce edificios de la primera línea litoral (González Pérez, 2012). Para poder llevar a cabo estos derribos, previamente fue necesaria la compra de los inmuebles por parte de la administración local. Adquisición que se pudo realizar gracias a los fondos provenientes del Estado (Pié et al., 2005). Existen otras opciones, gestionadas en el ámbito local, para poder ejecutar este tipo de intervenciones sin tener que depender de los recursos públicos del Estado, como podrían ser: la permuta de solares, la compra de edificios concretos o la adquisición de ciertos solares a cambio de permitir el aumento de edificabilidad en otros (las ya mencionadas transferencias de aprovechamiento).

Otra dinámica interesante y, a tener en cuenta a la hora de afrontar la problemática vinculada a la ausencia de residentes en determinadas épocas del año, es la que se está produciendo en algunos municipios del propio territorio valenciano, especialmente de la costa alicantina, en relación al proceso llamado "residencialización de inmigrantes climáticos". Esta dinámica es el resultado de la llegada de inmigrantes jubilados de países europeos que buscan unas condiciones climáticas más favorables, plasmadas en una mayor cantidad de horas de sol. Esto podría considerase como una alternativa más, aunque no debería ser la única, por las 
problemáticas a nivel social y de prestación de servicios que ya se está generando en determinadas zonas.

En definitiva, y en el caso de que sólo se propongan actuaciones dirigidas a la renovación como destino turístico, se debe evitar la realización de nuevas infraestructuras de transporte, pues son los elementos que incentivan la llegada de turistas y que a la larga hacen que se agote el modelo, como ya hemos indicado. Sin embargo, si se apuesta también por hacer ciudad, que es lo que nosotros defendemos, será imprescindible su ejecución para poder transformar y agrupar los distintos núcleos urbanos y las urbanizaciones aisladas (Pié et al., 2005).

\subsection{Las diferentes manifestaciones del Concepto de Ciudad sobre el espacio urbano}

Una vez argumentado que una de las alternativas de los municipios litorales valencianos pasaría por su transformación mediante actuaciones que los doten en mayor medida de las características de lo que hemos denominado Concepto de Ciudad, además de actualizar y renovar su oferta turística, se exponen a continuación los diferentes modelos de ciudad que se pueden obtener como resultado de dichas intervenciones. Además, bajo nuestro punto de vista, se defiende cuál debería ser el modelo a seguir, aunque como veremos posteriormente, algunas veces no será viable la implementación de ese modelo.

De manera genérica podemos identificar tres tipos de ciudad que cumplen con las características reseñadas como propias del Concepto de Ciudad: la Ciudad Pública del mundo clásico, donde se vive en el espacio público; la Ciudad Doméstica del mundo anglosajón, donde se hace vida en el interior de las viviendas; y la Ciudad Privada del mundo musulmán, donde la vida gira en torno al patio interior de la vivienda (Chueca Goitia, 1968). De estos tres modelos, los dos primeros (la Ciudad Pública y la Ciudad Doméstica) son los que encontramos entremezclados en las zonas urbanas europeas actuales, que son la base sobre la que proyectar la ciudad del futuro.

Ambos modelos de ciudad se podrían abordar a través de nueve parámetros, los cuales son opuestos entre sí. Seguidamente, se definen las características del modelo de Ciudad Pública, que es el modelo que postulamos como el objetivo a alcanzar en todos los casos en los que sea factible. Por tanto, las características del modelo de Ciudad Doméstica serán la antítesis de éstas (Tabla 3).

En primer lugar, prima la artificialidad del entorno urbano; es decir, deben prevalecer las edificaciones realizadas por el hombre sobre el elemento natural. Sin embargo, en la actualidad se hace también necesaria la introducción de espacios con vegetación que las estructuren debido al tamaño que las ciudades han adquirido. En segundo lugar, prevalece la continuidad/compacidad, lo que implica la inexistencia de discontinuidades en lo construido y la ausencia de elementos que generen límites físicos o psicológicos que restrinjan el desplazamiento por la trama urbana. En tercer lugar, el espacio se formaliza mediante su delimitación con lo construido y es percibido como una unidad. En cuarto lugar, se da una mezcla de usos (residencial, comercial, terciario, dotacional) tanto en los edificios como en la trama urbana, lo que genera actividades diversas realizadas por distintos grupos en diferentes horarios. 
En quinto lugar, el espacio público vertebra la ciudad y permite tanto los desplazamientos de personas y vehículos como la configuración de lugares que favorezcan la estancia en ellos. En sexto lugar, existe una fuerte relación visual entre las fachadas de los edificios y el espacio público que delimitan (concepto de Ventana a la calle). Esta relación se ve potenciada en las plantas bajas con comercios al favorecerse la permeabilidad y la transparencia, generando vida urbana y, de este modo, confiriendo seguridad a los transeúntes (Borja y Muxí, 2000). En séptimo lugar, tiene un carácter policéntrico, entendiendo como tal que las unidades estructurantes de la ciudad se encuentran repartidas por ésta y que prevalecen las de mediana y pequeña escala en detrimento de las grandes. En octavo lugar, se le da predominio al peatón de tal manera que la red viaria está supeditada a la peatonal para no generarle discontinuidades. En noveno y último lugar, la arquitectura (los edificios) está supeditada a los intereses generales de la ciudad. Las edificaciones satisfacen la homogeneidad espacial requerida pero sus fachadas están diseñadas para conferir diversidad al paisaje urbano. Sólo habrá algunas edificaciones que rompan esta tónica, convirtiéndose, al hacerlo, en elementos de referencia (Oliva i Casas, 2006).

Tabla 3: Comparación de las diferencias entre la Ciudad Pública y Ciudad Doméstica

\begin{tabular}{|l|l|l|}
\hline & \multicolumn{1}{|c|}{ Ciudad Pública } & \multicolumn{1}{c|}{ Ciudad Doméstica } \\
\hline 1 & Artificialidad & Introducción de vegetación entre los edificios \\
\hline 2 & $\begin{array}{l}\text { Continuidad y compacidad del espacio } \\
\text { construido }\end{array}$ & Discontinuidad del espacio construido \\
\hline 3 & $\begin{array}{l}\text { Espacio público acotado, delimitado, } \\
\text { modelado y percibido como unidad }\end{array}$ & Espacio público no delimitado \\
\hline 4 & Mezcla de usos & Usos separados \\
\hline 5 & Espacio público vertebrador & Espacio público residual y amorfo \\
\hline 6 & Relación de las viviendas con la calle & Dilución de la percepción de la calle \\
\hline 7 & $\begin{array}{l}\text { Unidades estructurantes de pequeña o } \\
\text { mediana escala repartidas por la zona urbana }\end{array}$ & $\begin{array}{l}\text { Unidades de gran escala que se especializan } \\
\text { en un solo uso }\end{array}$ \\
\hline 8 & Prioridad del peatón & Prioridad del Coche \\
\hline 9 & Arquitectura supeditada al urbanismo & Protagonismo de la arquitectura \\
\hline
\end{tabular}

Fuente: Elaboración propia a partir de Oliva i Casas (2006).

Llegados a este punto, no debemos dejar de puntualizar, aunque pueda resultar obvio, que el modelo de ciudad por el que se apuesta, la Ciudad Pública, es a la vez el más sostenible, razón que le es intrínseca a las características básicas que lo definen, de las cuales destacan elementos fundamentales de las consideradas ciudades sostenibles. Características como las de continuidad, compacidad, creación de espacios públicos, mezcla de usos, espacio público vertebrador o la priorización del protagonismo del peatón tienen una directa y clara relación con los conceptos de habitabilidad y movilidad urbana (Borja y Muxí, 2000; Folch, 2003; Cals y Bosch, 2005; Esteban, 2006; Oliva i Casas, 2006; Serrano Rodríguez, 2006; Cortés Chavez, 2009; Fariña y Naredo (Dir.), 2010; y Rueda et al., 2012). 


\section{VIABILIDAD DE APLICACIÓN DEL CONCEPTO DE CIUDAD EN LAS DISTINTAS CARACTERÍSTICAS URBANAS}

Las posibilidades de otorgar las características de lo que hemos venido a denominar Concepto de Ciudad a los municipios turísticos del litoral valenciano estarán en función de dos variables fundamentales: por un lado, las propias características del espacio urbano que se quiere transformar, $y$, por otro lado, el periodo de tiempo en el que se quiera conseguir dicho objetivo (corto, medio o largo plazo).

Las intervenciones necesarias para dotar al municipio turístico de las características del Concepto de Ciudad de las que éste es carente necesitan ejecutarse durante un prolongado periodo de tiempo, por lo que el resultado final de la transformación generalmente se alcanza en el medio-largo plazo. Por otro lado, también hay que tener en cuenta el modelo de ciudad a alcanzar con las intervenciones, pues los dos modelos (la Ciudad Pública y la Ciudad Doméstica), como se ha expuesto en el apartado anterior, son antagónicos entre sí. Por esta razón, tiene tanta relevancia las características del núcleo urbano existente a la hora de planificar las intervenciones. Nosotros defendemos la realización de aquellas que promuevan la transformación del municipio turístico en Ciudad Pública. Sin embargo, si el modelo tomado como referencia para su desarrollo urbano fue el de la Ciudad Doméstica, será muy compleja la substitución del modelo de ciudad al tener características opuestas.

Además de los nueve parámetros en los que los dos modelos de ciudad se contraponen, ambos modelos también se diferencian por las características urbanas que en ellas se utiliza. Respecto de estas características, existen tres tipos distintos: el compacto, el disperso y el mixto.

El compacto es aquel que presenta una alta concentración poblacional, distribuida por un territorio acotado en superficie; mientras que el disperso es su opuesto; es decir, aquél que presenta una densidad reducida porque la población está asentada en un espacio muy amplio. En nuestra opinión, el modelo disperso es peor, y mucho menos sostenible, ya que exige consumir una gran cantidad de territorio además de que tiende a eliminar la razón de ser embrionaria de las ciudades: "ser un lugar para el encuentro y el intercambio (comercial, cultural, social, etc.)" (Folch, 2003, p. 29). Por último, está el tipo mixto, que se caracteriza por tener una alta compacidad y mezclar usos, tanto a la escala de un edificio (edificio de viviendas con comercio en la planta baja u oficinas en las primeras plantas), como en el ámbito de barrio (donde, además, aparecen distintas tipologías edificatorias en una misma zona urbana, como pueden ser viviendas unifamiliares, edificios de viviendas, edificios terciarios, etc.) (Folch, 2003). El tipo mixto, que es la evolución del tipo compacto mediante la introducción de diversidad y complejidad, se corresponde con el modelo de Ciudad Pública, mientras que el tipo disperso es el que encontramos en la Ciudad Doméstica.

Seguidamente, se analizan las características que podemos encontrar en los municipios del litoral mediterráneo para identificar la viabilidad de transformar el modelo de Ciudad Doméstica en el modelo de Ciudad Pública.

Los núcleos históricos mediterráneos valencianos se caracterizan, en lo general, por ser de tipo compacto o por el mixto, en los casos en los que hayan adquirido una cierta relevancia 
en el territorio como polo de actividades (Nolasco, 2015). Los crecimientos a partir de éstos, motivados por el turismo, se realizaron con una tipología urbana compacta (con una alta compacidad edificatoria pero únicamente con el uso residencial) o por el disperso (caracterizado por una baja densidad de población repartida en una vasta superficie de territorio). Así pues, para aproximarnos al Concepto de Ciudad Pública habrá que realizar intervenciones que cambien la tipología urbana compacta y dispersa a mixta. En el caso del compacto, únicamente hará falta introducir nuevos usos (comercial y dotacional) y relacionarlos con el uso residencial existente. Sin embargo, existirá una gran dificultad para transformar la tipología urbana dispersa en mixta, pues su característica respecto a la densidad es completamente opuesta y requerirá no sólo una introducción de nuevos usos sino también una densificación del tejido urbano existente.

En este segundo supuesto (existencia de la tipología dispersa), la dificultad de esa densificación para orientarlo hacia la tipología de la Ciudad Pública dependerá de la tipología edilicia utilizada. Si está configurada por vivienda colectiva con servicios aparejados y con espacio libre circundante de uso privado, las actuaciones se realizarían sobre dicho espacio; mientras que, si está conformada por una urbanización de viviendas unifamiliares, la densificación implicaría la substitución de algunas de estas viviendas por tipologías plurifamiliares de bloques o torres.

Pero, en el caso hipotético de que se tuviera éxito con esta compleja actuación, con ella sólo obtendríamos el carácter urbano del modelo de Ciudad Pública y aún se deberían cumplir el resto de los nueve parámetros relacionados. Por lo que las intervenciones a desarrollar se deberían insertar en una actuación global que tuviera como objetivo a largo plazo el alcanzar todas o el mayor número de esos parámetros. Sin embargo, en los casos de urbanizaciones de viviendas unifamiliares diseminadas por el territorio, cuya morfología y fisonomía es la antítesis de la ciudad tradicional, es casi imposible reconvertirlas en Ciudad. Es por ello que, bajo nuestro punto de vista, a la hora de afrontar la mejora de estos desarrollos urbanos dispersos, habrá que realizar intervenciones que los doten del mayor número de las características del Concepto de Ciudad, descartando cambiar el modelo en el que se inspiran. De este modo, con la ejecución de estas intervenciones sobre el tejido urbano disperso, nos aproximaríamos a la obtención de la Ciudad Doméstica que es el modelo que, de un modo enormemente desvirtuado, fue tomado como referencia en los municipios turísticos mediterráneos.

En la Tabla 4 se han identificado las distintas tipologías urbanas que se encuentran en los municipios del litoral mediterráneo valenciano en función de dos variables: la localización del núcleo tradicional (en la costa o en el interior) y la expansión urbana de éste (en continuidad con lo construido o ex novo). Posteriormente, se han indicado los diferentes parámetros que se pueden encontrar para cada uno de los tipos y se han relacionado con el modelo de ciudad que es la referencia para dicho carácter urbano. Finalmente, en la última columna se han indicado las distintas características respecto al Concepto de Ciudad de las que pueden carecer cada uno de los tipos. Es por ello que, en este cuadro, se representan las posibles carencias teóricas que se deberían subsanar en cada uno de los tipos para obtener de facto el modelo de ciudad que se ha tomado como referencia para su desarrollo. 
Tabla 4: Parámetros urbanos utilizados en función de los crecimientos urbanos del municipio turístico mediterráneo

\begin{tabular}{|c|c|c|c|c|}
\hline $\begin{array}{c}\text { Partes que configuran } \\
\text { un municipio turístico } \\
\text { mediterráneo }\end{array}$ & $\begin{array}{l}\text { Carácter } \\
\text { urbano }\end{array}$ & $\begin{array}{l}\text { Referencia del } \\
\text { Modelo de } \\
\text { ciudad }\end{array}$ & $\begin{array}{c}\text { Cumple } \\
\text { Concepto de } \\
\text { Ciudad }\end{array}$ & $\begin{array}{c}\text { Características } \\
\text { de las que puede } \\
\text { carecer }\end{array}$ \\
\hline \multirow{2}{*}{$\begin{array}{l}\text { Núcleo histórico fundado en } \\
\text { contacto con la costa }\end{array}$} & Compacto & Ciudad Pública & No & $3 ; 6$ \\
\hline & Mixto & Ciudad Pública & $\mathrm{Si}$ & \\
\hline $\begin{array}{l}\text { Núcleo histórico secundario } \\
\text { en contacto con la costa }\end{array}$ & Compacto & Ciudad Pública & No & $2 ; 3 ; 4 ; 6$ \\
\hline \multirow{2}{*}{$\begin{array}{l}\text { Núcleo histórico en el interior } \\
\text { del litoral }\end{array}$} & Compacto & Ciudad Pública & No & $3 ; 6$ \\
\hline & Mixto & Ciudad Pública & $\mathrm{Si}$ & \\
\hline \multirow{3}{*}{$\begin{array}{l}\text { Crecimiento vinculado al } \\
\text { núcleo existente (en el } \\
\text { interior o en el litoral) }\end{array}$} & Compacto & Ciudad Pública & No & $3 ; 5 ; 6$ \\
\hline & Mixto & Ciudad Pública & $\mathrm{Si}$ & \\
\hline & Disperso & \begin{tabular}{|l|} 
Ciudad \\
Doméstica \\
\end{tabular} & No & $1 ; 2 ; 3 ; 4 ; 5 ; 6 ; 7$ \\
\hline \multirow{2}{*}{$\begin{array}{l}\text { Núcleo ex novo no vinculado } \\
\text { con núcleos existentes }\end{array}$} & Compacto & Ciudad Pública & No & $1 ; 2 ; 3 ; 4 ; 5 ; 6 ; 7$ \\
\hline & Disperso & $\begin{array}{l}\text { Ciudad } \\
\text { Doméstica }\end{array}$ & No & $1 ; 2 ; 3 ; 4 ; 5 ; 6 ; 7$ \\
\hline
\end{tabular}

Elaboración propia.

\section{CONCLUSIONES}

Los municipios rurales del litoral mediterráneo valenciano estuvieron fuertemente influenciados por la irrupción del turismo a mediados del pasado siglo XX. Esta nueva actividad económica, que expulsó a las actividades tradicionales por su mayor rentabilidad, tuvo una gran incidencia sobre la evolución de estos municipios. El gran crecimiento que la mayoría de ellos experimentaron fue provocado porque supeditaron en gran medida sus decisiones a satisfacer las necesidades y exigencias de la demanda turística, la cual, originariamente, consistía en el mero proceso de alojar el mayor número de visitantes posible. Por ello, este crecimiento se realizó mediante la construcción y venta de viviendas que proveyeran de alojamiento a los residentes temporales y a los turistas, conformando de este modo, zonas residenciales monofuncionales. Así pues, este modo de actuar hizo, en términos generales, que estos municipios dejaran de lado, en parte, las necesidades de los habitantes, la estructura urbana que organiza el municipio y la relación con el entorno circundante, y se convirtieran en destinos turísticos.

Sin embargo, este modelo de desarrollo en el que se sustentan dichos municipios los encamina, en sus distintas etapas de evolución, hacia una pérdida progresiva de su atractivo turístico. De este modo, el municipio turístico puede verse abocado, en el medio y largo plazo, a su estancamiento y posible declive como destino para turistas.

Por ello, el municipio turístico debe estar continuamente renovándose para no perder su atractivo frente a otros competidores. A pesar de ello, se tiene que insistir en las dificultades 
que impone el modelo predominante, con una gran cantidad de edificios residenciales que permanecen vacíos e infrautilizados durante los meses no estivales (dos tercios del año). Esto se debe principalmente a tres causas: en primer lugar, a que se carece de los estándares mínimos de espacio público y de dotaciones para la población potencial que puede ser alojada; en segundo lugar, a que se trata de viviendas de segunda residencia y, en tercer lugar, a la fuerte estacionalidad del producto turístico ofertado.

Ante esta realidad descrita, se han tratado distintas opciones de intervención existentes -especialmente en el ámbito de la teoría- para intentar evitar esas situaciones de declive indicadas. A partir de este planteamiento, se ha defendido como una buena alternativa la de revitalizar los municipios turísticos para intentar transformarlos conforme a las principales características que identifican al Concepto de Ciudad, e incorporar nuevas actividades económicas que los diversifiquen para atraer, en parte, mayor población residente permanente. Con esta propuesta de intervención, con gran importancia en el ámbito construido, no sólo se podría renovar su atractivo turístico, sino que se mejoraría el espacio urbano y, al mismo tiempo, las condiciones de vida de los residentes.

A pesar de ello, la traslación de dicha propuesta de intervención sobre los municipios, y los resultados que de ella se obtengan, estará en función del modelo de ciudad (Pública o Doméstica) tomado como referencia para su desarrollo y el carácter urbano (Compacto, Mixto o Disperso) que le es propio a cada modelo. Ambos modelos de ciudad son antagónicos y cada uno de ellos se corresponde con un carácter urbano distinto.

Defendemos que el mejor modelo urbano y el más sostenible es el de la Ciudad Pública, que tiene un patrón mixto y que se caracteriza por: primar el elemento artificial (la zona construida) desarrollado de manera continua, formalizando y delimitando, por contraste, el espacio público que dota de estructura al tejido urbano; promover la mezcla de usos urbanos distribuidos uniformemente en su interior mediante unidades de pequeña o mediana escala; propiciar la relación visual entre los edificios y el espacio público que confiere seguridad; empoderar al peatón; y primar el conjunto urbano sobre el edificio singularizado. Sin embargo, algunas de las expansiones urbanas de los municipios turísticos del litoral se han llevado a cabo con un carácter disperso, al haberse tomado como referente el modelo de Ciudad Doméstica, y por ello será difícil modificar ese modelo, ya que no bastará con intervenir en el espacio público y sobre algunos edificios residenciales puntuales, sino que será necesario también actuar sobre el conjunto edificado para aumentar la densidad urbana y modificar el modo en el que las construcciones se relacionan con el espacio público, con la problemática social y económica que esto comportaría.

Es decir, para intentar transformar el modelo, sería necesario sustituir las tipologías de viviendas unifamiliares y edificios aislados en parcela por otras que delimitaran el espacio público y favorecieran la función comercial a nivel de calle, lo que es muy complejo, precisa de mucho tiempo y exige gran voluntad política. Por tanto, en estos últimos casos, en los que es de gran dificultad el cambiar el modelo de ciudad utilizado, las intervenciones deberían centrarse únicamente en dotar a los municipios de las características que identifican el Concepto de Ciudad con el objetivo de mejorar estos espacios urbanos y, en definitiva, la atracción del destino turístico. 


\section{REFERENCIAS BIBLIOGRÁFICAS}

Aledo, A. (2008). De la tierra al suelo: la transformación del paisaje y el nuevo turismo residencial, Arbor. Ciencia, Pensamiento y Cultura, 184 (729), 99-113.

Aledo, A., Mazón, T. y Mantecón, A. (2007). La insostenibilidad del turismo residencial. En D. Lagunas (coord.): Antropología y turismo: claves culturales y disciplinares (pp. 185208). Alicante: Plaza y Valdés.

Ball-Ilosera, M. y Albó, A. (2005). Costa Brava: entre el mite i el cinisme. In Debat Costa Brava. Congrés: un futur sostenible (pp. 24-27). Col-legi Oficial d'Arquitectes de Catalunya de Girona.

Barragán Muñoz, J. M. (1993). Ordenación, planificación y gestión del espacio litoral. Barcelona: Oikos-Tau.

Blanco, R. (2012). Planificación de destinos turísticos maduros. Consideraciones para su reconversión. En F. Vera Rebollo y I. Rodríguez Sánchez (eds.), Renovación y reestructuración de destinos turísticos en áreas costeras: Marco de análisis, procesos, instrumentos y realidades (pp. 55-68). Universitat de València.

Blanquer, B., Payá, J. A., Escribano, A., García-Lliberós, M. y Marqués, J. V. (1983). Mesa redonda: la vivienda ilegal clandestina de segunda residencia en medio rural. En Urbanismo y Medio Rural. Valencia La vivienda ilegal de segunda residencia (pp. 97-110). Diputación de Valencia.

Borja, J. y Muxí, Z. (2000). El espacio público, ciudad y ciudadanía, Barcelona.

Busquets, J. (2003). Les formes urbanes del litoral català. Diputació de Barcelona.

Busquets, J. (2004). Comprendre el territorio per dissenyar-lo, Papers, Regió Metropolitana de Barcelona 41, 9-23.

Butler, R.W. (1980). The concept of a tourist area cycle of evolution: implications for management of resources, The Canadian Geographer, XXIV, I, 5-12.

Calduch, J. (2001). Vacaciones i arquitectura. Transitando, recorriendo, bordeando los márgenes. En J. Granell i March (coord.), La arquitectura del Sol (pp.92-105). Barcelona: Col·legi d'Arquitectes de Catalunya.

Callizo Soneiro, J. (1991). Aproximación a la geografía del turismo, Espacios y sociedades. Serie general 21. Madrid: Síntesis.

Cals, J. y Bosch, C. (2005). Presentació de les conclusions. En Debat Costa Brava. Congrés: un futur sostenible (pp. 374-379). Col-legi Oficial d'Arquitectes de Catalunya de Girona.

Chiner Vives, J. J. (2006). Benidorm. Los orígenes de la ciudad vertical. Ajuntament de Benidorm.

Chueca Goitia, F. (1968). Breve historia del urbanismo. Madrid: Alianza.

Cortés Chavez, S. (2009). La capacidad de carga como herramienta para la ordenación sostenible del territorio, Cuaderno de Investigación Urbanística 65, 35-56.

Donaire, J. A. (2005). La lògica espacial del turisme de la Costa Brava. In Debat Costa Brava. Congrés: un futur sostenible (pp. 218-225). Col-legi Oficial d'Arquitectes de Catalunya de Girona.

Donaire, J. A. y Mundet, LI. (2002). Estrategias de reconversión turística de los municipios litorales catalanes. In P. Pumares et al. (Coord), Turismo y transformaciones urbanas en el siglo XXI (pp. 39-49). Universidad de Almería. 
Esteban, J., Ribera, J., Martí, J., Sargatal, J., Biosca, D. y Fuses, J. (2005). Taula rodona. In Debat Costa Brava. Congrés: un futur sostenible (pp. 122-135). Col·legi Oficial d’Arquitectes de Catalunya de Girona.

Esteban, J. (2006). La necesaria contención de la dispersión urbana. En A. Tarroja y R. Camagni (Coord.), Una nueva cultura del territorio. Criterios sociales y ambientales en las políticas y el gobierno del territorio (pp. 267-277). Diputació de Barcelona.

Fariña Tojo, J. y Naredo, J. M. (Dir.) (2010). Libro Blanco de la Sostenibilidad en el Planeamiento Urbanístico Español. Madrid: Ministerio de Vivienda, Gobierno de España.

Fernandez Tabales, A. (2004). Turismo y Ordenación del Territorio, Quaderns de Política Econòmica, Revista Electronica, Segunda Epoca, no 7, 35-47.

Ferrandis, A. y Noguera, J. (2016). Planeamiento territorial sostenible: un reto para el futuro de nuestras sociedades; criterios aplicados. En Cadernos Metropole, São Paulo, v. 18, n. 37, pp. 743-763.

Ferrer Marsal, J. (2002). Construir la Costa, el litoral Valenciano. Valencia: Consell Valencià de Cultura.

Folch, R. (2003). Los conceptos socioecológicos de partida. En R. Folch. (coord.), El territorio como sistema: conceptos y herramientas de ordenación (pp. 19-42). Diputació de Barcelona.

Gaja, F. (2008). El «tsunami urbanizador» en el litoral mediterráneo. El ciclo de hiperproducción inmobiliaria 1996-2006, Scripta Nova, Vol. XII, num. 270 (66).

Gaja, F. (2012). Antes, durante y después del tsunami inmobiliario en el País Valenciano. En F. Gaja (Ed.), DeCOASTruction: la desconfiguración del litoral mediterráneo español (pp. 78-126). Universitat Politècnica de València.

García-Delgado, C. (2002). Vivir del turismo, morir del turismo. En J. Granell i March (coord.), La arquitectura del Sol (pp. 174-178). Barcelona: Col-legi d'Arquitectes de Catalunya.

García Nart, M., Llauger, M. A. y Socias, M. (1991). El proceso de planificación y los desarrollos de segunda residencia. En Desarrollos de segunda residencia, Coloquio internacional. Palma de Mallorca, 4-7 junio 1990 (pp. 201-214). Madrid: Ministerio de Obras Públicas y Transportes.

González Pérez, J. M. (2012). Un plan nuevo para un núcleo turístico maduro. Reconversión y rehabilitación de la Platja de Palma (Mallorca). En F. Vera Rebollo y I. Rodríguez Sánchez (eds.), Renovación y reestructuración de destinos turísticos en áreas costeras: Marco de análisis, procesos, instrumentos y realidades (pp. 243-268). Universitat de València.

Iribas, J. M. (1997). Benidorm: Manual de uso. En Vía Arquitectura no1: Arquitectura en la costa (pp. 66-73). Valencia: COACV.

Mantecón, A. (2005). La construcción social de la experiencia turística: El caso de los anfitriones. En T. Mazón y A. Aledo (coord.), Turismo residencial y cambio social: nuevas perspectivas teóricas y empíricas (pp. 391-410). Alicante: CAM Obras Sociales.

Mazón, T. y Aledo, A. (2005). El dilema del turismo residencial: ¿Turismo o desarrollo inmobiliario? En T. Mazón y A. Aledo (coord.), Turismo residencial y cambio social: nuevas perspectivas teóricas y empíricas (pp. 13-30). Alicante: CAM Obras Sociales.

Navarro Jurado, E. (2012). La Costa del Sol Occidental y el Plan Qualifica, ¿renovación de un destino maduro? En F. Vera Rebollo y I. Rodríguez Sánchez (eds.), Renovación y reestructuración de destinos turísticos en áreas costeras: Marco de análisis, procesos, instrumentos y realidades (pp. 201-217). Universitat de València. 
Nolasco Cirugeda, A. (2015). La dimensión Urbana de la Costa Blanca; Ocupación, espacio y complejidad en Dénia, Benidorm y Torrevieja. Tesis Doctoral, Universidad de Alicante, $342 \mathrm{pp}$.

Obiol, E. M. y Pitarch, M. D. (2011). El litoral turístico valenciano. Intereses y controversias en un territorio tensionado por el residencialismo. Boletín de la Asociación de Geógrafos Españoles 56, (pp. 177-200).

Obiol, E.M. y Ferrandis, A. (2011). Gandia y sus prácticas de Renovación Turística. En F. Vera Rebollo y I. Rodríguez Sánchez (eds.), Renovación y reestructuración de destinos turísticos en áreas costeras: Marco de análisis, procesos, instrumentos y realidades (pp. 375-398). Universitat de València.

Oliva i Casas, J. (2006). Reflexions entorn de la ciutat. Barcelona: Fundació Ramon Trias Fargas. Perelli, O. (2012). La reconversión de los destinos maduros del litoral en el actual contexto de cambio global: una reflexión sobre nuevos instrumentos para su impulso. En F. Vera Rebollo y I. Rodríguez Sánchez (eds.), Renovación y reestructuración de destinos turísticos en áreas costeras: Marco de análisis, procesos, instrumentos y realidades (pp. 37-54). Universitat de València.

Perlado, S. y Elorrieta, I. (coord.) (2007). Libro verde del Medio Ambiente Urbano. Tomo I. Madrid: Ministerio de Medio Ambiente, Gobierno de España.

Pesci, R. (2003). El urbanismo y la cultura ambiental. En R. Folch. (coord.), El territorio como sistema: conceptos y herramientas de ordenación (pp. 101-119). Diputació de Barcelona.

Pié, R. (2005). Ara toca fer ciutat. En Debat Costa Brava. Congrés: un futur sostenible (pp. 50-87). Col-legi Oficial d'Arquitectes de Catalunya de Girona.

Pié, R., Crespo, X., Hostench, A., Donés, J., Oliver, J. Ball-Ilosera, M. (2005). Taula Rodona. En Debat Costa Brava. Congrés: un futur sostenible (pp. 354-371). Col-legi Oficial d'Arquitectes de Catalunya de Girona.

Pié, R. y Barba, R. (1996). Segunda residencia y turismo versus residencia permanente. En R. Barba y Pié, R. (Eds.), Arquitectura y turismo: planes y proyectos (pp. 43-48). Barcelona: Universitat Politècnica de Catalunya y Secretaría General de Turismo.

Pitarch, M. D. (2009). Los recursos territoriales turísticos: su gestión y organización en entornos locales. En J. Noguera et al. (Coord.), Gestión y promoción del desarrollo local (pp. 445-476). Universitat de València.

Ponce Herrero, G. (2006). La fragmentación de la forma urbana en la Comunidad Valenciana. En G. Ponce Herrero (ed.), La ciudad fragmentada: nuevas formas de hábitat (pp. 89-129). Universidad de Alicante.

Priestley, C. K. (1996). Evolución turística y repercusiones espaciales: medidas correctoras hacia la sostenibilidad. En R. Barba y R. Pié (Eds.), Arquitectura y turismo: planes y proyectos (pp. 99-110). Barcelona: Universitat Politècnica de Catalunya y Secretaría General de Turismo.

Ramírez, J. (1983). La crisis de la ciudad moderna, la vivienda de segunda residencia y las ilegales. En Urbanismo y Medio Rural. Valencia La vivienda ilegal de segunda residencia (pp. 29-39). Diputación de Valencia.

Rovira Soto, M. T. y Anton Clavé, S. (2014). De destino a ciudad. La reformulación urbana de los destinos turísticos costeros maduros. El caso de la Costa Daurada central. ACE: Architecture, City and Environment 25, pp. 373-392. 
Rueda, S., De Cáceres, R., Cuchí, A., y Brau, Li. (2012). El Urbanismo Ecológico: Su aplicación en el diseño de un ecobarrio en Figueres. Barcelona: Agència d'Ecologia Urbana de Barcelona.

Sánchez Cabrera, J.V. (2011). La Gestión Integral de las Zonas Costeras: Un balance para la Comunitat Valenciana 1956-2010. En Farinós, J. (coord.): La gestión integrada de zonas costeras (pp. 219-240). Universitat de València.

Santatecla, J. (2015). Desarrollo urbano de la playa norte de Gandia. En J. J. Tuset y R. Temes (Coord.), Territorio litoral: orilla marítima (pp. 122-127). Valencia: General de Ediciones de Arquitectura.

Serrano Rodríguez, A. (2006). La aplicación de los principios de la ETE en la ordenación del territorio en España. ¿Hacia un modelo policéntrico y sostenible? En A. Tarroja R. Camagni (Coord.), Una nueva cultura del territorio. Criterios sociales y ambientales en las políticas y el gobierno del territorio (pp. 69-106). Diputació de Barcelona.

Simancas, M. R. (2012). Evaluando políticas públicas de renovación de destinos turísticos maduros: el proceso de reconversión turística de Canarias. En F. Vera Rebollo y I. Rodríguez Sánchez (eds.), Renovación y reestructuración de destinos turísticos en áreas costeras: Marco de análisis, procesos, instrumentos y realidades (pp. 163-199). Universitat de València.

Tatjer Mir, M. (2009). En los orígenes del turismo litoral: los baños de mar y los balnearios marítimos en Cataluña. Scripta Nova. Revista Electrónica de Geografía y Ciencias Sociales. Barcelona: Universidad de Barcelona, vol. XIII, núm. 296 (5).

Torres Alfosea, F. J. (1997). Ordenación del litoral de la costa blanca. Universidad de Alicante.

Tuset, J. J. y Temes, R. (2015). Estrategias para la regeneración sostenible de asentamientos turísticos en la costa mediterránea. En J. J. Tuset y R. Temes (Coord.), Territorio litoral: orilla marítima (pp. 144-149). Valencia: General de Ediciones de Arquitectura.

Vera Rebollo, J. F. (1996). La variable territorial en los procesos de desarrollo turístico. En R. Barba y Pié, R. (Eds.), Arquitectura y turismo: planes y proyectos (pp. 87-98). Barcelona: Universitat Politècnica de Catalunya y Secretaría General de Turismo.

Vera Rebollo, J. F. y Baños Castiñeira, C. J. (2010). Renovación y reestructuración de los destinos turísticos consolidados del litoral: las prácticas recreativas en la evolución del espacio turístico. Boletín de la Asociación de Geógrafos Españoles 53, pp. 329-353.

Vera Rebollo, J. F. y Ivars, J. A. (2002). Turismo, territorio y desarrollo regional en la Comunidad Valenciana. En D. Blanquer Criado (dir.), Ordenación y gestión del territorio turístico (pp. 149-189). Valencia: Tirant lo Blanch.

Vera Rebollo, J. F. (coord.), López Palomeque, F., Marchena, M. J. y Anton Clavé, S. (2013). Análisis territorial del turismo y planificación de destinos turísticos. Valencia: Tirant Humanidades.

\section{CONTRIBUCIONES DE LAS/LOS AUTORES/RES:}

Los autores firmantes del presente artículo, declaran que han participado de forma coordinada y por igual en cada una de las labores dedicadas a: a) la concepción original del trabajo; b) al análisis, adquisición e interpretación de los datos; c) la redacción y revisión crítica del contenido; y d) la aprobación final de la versión a publicar. 